\title{
Human Cardiac Ischemia-Reperfusion Injury: Blunted Stress Response with Age
}

\author{
Ashley Oliveira ${ }^{1}$, Bobby Yanagawa ${ }^{2}$, Adrian Quan ${ }^{3}$, Subodh Verma ${ }^{2}$, and David Hood ${ }^{1}$ \\ ${ }^{1}$ York University Faculty of Health \\ ${ }^{2}$ University of Toronto Faculty of Medicine \\ ${ }^{3}$ St Michael's Hospital Keenan Research Centre for Biomedical Science
}

March 17, 2021

\begin{abstract}
Background \& Aim: Autophagy is a cytoprotective recycling mechanism, capable of digesting dysfunctional cellular components, and this process is associated with pro-survival outcomes. Autophagy may decline in the aging myocardium, thereby contributing to cardiac dysfunction. However, it remains to be established how autophagy responds to ischemia-reperfusion stress with age. Methods: Samples from the right atrium were collected from young ([?]50 years; $\mathrm{n=5}$ ) and aged ([?]70 years; $\mathrm{n}=11$ ) patients prior to and immediately following cardioplegic arrest during coronary artery bypass grafting (CABG) surgery, a model of human ischemia-reperfusion injury. Results: Mitochondrial content did not differ between the age groups, however a $32 \%$ reduction in UQCRC2 ( 0.74 vs 0.53 , effect of age, $\mathrm{p}=0.03$ ) was seen with age, indicating possible compositional disruptions. In response to IR, VDAC (0.75 vs $1.05, \mathrm{p}=0.03$ ) and COX-I protein (0.63 vs $1.10, \mathrm{p}=0.03$ ) was over expressed in young, but not in aged patients. Reductions in Parkin ( 0.95 vs 0.49 , interaction effect, $\mathrm{p}=0.04)$ and NIX (0.60 vs $0.21, \mathrm{p}=0.004)$ protein expression with age suggest an impairment in mitochondrial recycling, which may lead to an accumulation of dysfunctional mitochondria. Following IR, our data suggest that in the young cohort, autophagy is reduced as a Beclin-1 decreased by $63 \%$ (0.95 vs 0.36 , $\mathrm{p}=0.001$ ) and no changes were observed in either p62 or LC3-II:I ratio. Conclusion: Our data demonstrate a blunted cardiac mitochondrial response to ischemia with age, accompanied by a possible impairment in mitophagy. These findings support an age-associated inability of the atrial myocardium to mount appropriate adaptive responses to stress.
\end{abstract}

Human Cardiac Ischemia-Reperfusion Injury:

Blunted Stress Response with Age

Ashley N. Oliveira, $\mathrm{MSc}^{1}$, Bobby Yanagawa, MD, $\mathrm{PhD}^{2,3}$, Adrian Quan, MPhil ${ }^{2}$, Subodh Verma, MD, PhD $2,3,4$ \& David A. Hood, PhD $^{1}$

${ }^{1}$ Muscle Health Research Center, York University, Toronto, ON, Canada M3J 1P3

${ }^{2}$ Division of Cardiac Surgery, Keenan Research Centre for Biomedical Science of St. Michael's Hospital, Toronto, ON, Canada M5B 1W8.

Departments of ${ }^{3}$ Surgery and ${ }^{4}$ Pharmacology and Toxicology, University of Toronto, Toronto, ON, Canada M5S 1A1

Keywords: Mitochondria, mitophagy, autophagy, ischemia-reperfusion, aging myocardium

Running Head: Mitochondria in Aged Heart

Funding: This work was supported by the Canadian Institutes for Health and Research.

To whom correspondence should be addressed: David A. Hood, PhD 
Muscle Health Research Centre,

York University, 4700 Keele St, Toronto, ON, M3J 1P3, Canada Tel: (416) 736-2100 ext.66640 Email: dhood@yorku.ca

Abstract

Background \& Aim: Autophagy is a cytoprotective recycling mechanism, capable of digesting dysfunctional cellular components, and this process is associated with pro-survival outcomes. Autophagy may decline in the aging myocardium, thereby contributing to cardiac dysfunction. However, it remains to be established how autophagy responds to ischemia-reperfusion stress with age.

Methods: Samples from the right atrium were collected from young ([?]50 years; $n=5)$ and aged ([?]70 years; $\mathrm{n}=11$ ) patients prior to and immediately following cardioplegic arrest during coronary artery bypass grafting (CABG) surgery, a model of human ischemia-reperfusion injury.

Results: Mitochondrial content did not differ between the age groups, however a $32 \%$ reduction in UQCRC2 ( 0.74 vs 0.53 , effect of age, $\mathrm{p}=0.03$ ) was seen with age, indicating possible compositional disruptions. In response to IR, VDAC (0.75 vs $1.05, \mathrm{p}=0.03)$ and COX-I protein (0.63 vs $1.10, \mathrm{p}=0.03)$ was over expressed in young, but not in aged patients. Reductions in Parkin ( 0.95 vs 0.49 , interaction effect, $\mathrm{p}=0.04$ ) and NIX ( 0.60 vs $0.21, p=0.004)$ protein expression with age suggest an impairment in mitochondrial recycling, which may lead to an accumulation of dysfunctional mitochondria. Following IR, our data suggest that in the young cohort, autophagy is reduced as a Beclin- 1 decreased by $63 \%$ ( 0.95 vs $0.36, \mathrm{p}=0.001$ ) and no changes were observed in either p62 or LC3-II:I ratio.

Conclusion: Our data demonstrate a blunted cardiac mitochondrial response to ischemia with age, accompanied by a possible impairment in mitophagy. These findings support an age-associated inability of the atrial myocardium to mount appropriate adaptive responses to stress.

Abbreviations

\begin{tabular}{ll}
\hline CABG & Coronary artery bypass grafting \\
\hline COX-I & Cytochrome c oxidase subunit I \\
COX-IV & Cytochrome c oxidase subunit IV \\
HRP & Horseradish peroxidase \\
Hsp70 & Heat shock protein 70 \\
IR & Ischemia-reperfusion \\
LC3 & Microtubule-associated proteins 1A/1B light chain 3B \\
NIX & Nip3-like protein X \\
P62 & Sequestosome 1 \\
SDS-PAGE & Sodium dodecyl sulfate-polyacrylamide gel electrophoresis \\
TFE3 & Transcription factor E3 \\
TFEB & Transcription factor EB \\
UQCRC2 & Cytochrome b-c1 complex subunit 2 \\
V-ATPase & Vacuolar-type ATPase \\
VDAC & Voltage-dependent anion channel \\
\hline
\end{tabular}

\section{Introduction}

Aging has significant structural, metabolic and biochemical repercussions on a variety of tissues, including the heart. Many age-associated changes converge on mitochondria, which include disrupted morphology 
characterized by swollen organelles ${ }^{1}$, and functional impairments including declines in oxidative phosphorylation and elevations in reactive oxygen species (ROS) emission ${ }^{2}$. Mitochondrial maintenance throughout the lifespan is critical for cardiac health, which relies on the removal and degradation of dysfunctional mitochondria via the lysosomes through a selective form of autophagy, known as mitophagy.

Autophagy is a cytoprotective mechanism, and is often associated with pro-survival outcomes in the face of cellular stress, including ischemia-reperfusion (IR) insults ${ }^{3-7}$. Autophagy provides a pivotal step in determining cell fate, as damaged mitochondria that do not undergo mitophagy may initiate apoptosis or cell death $^{8,9}$. It has been reported that autophagy decreases with age and this is thought to contribute to the aging phenotype ${ }^{10}$. The importance of autophagy in the heart has been demonstrated through loss- and gain-of-function animal models in which impaired autophagy has resulted in cardiomyopathy ${ }^{11,12}$, while enhanced autophagy has promoted longevity ${ }^{13}$. Therefore, it is important to understand how the intersection of age impacts the ability of the heart to respond to stress and injury, which in turn may allow a more tailored approach for interventions and therapies.

To better understand the role of age in implementing a pro-survival stress response in the heart, samples of the right atrial appendage were taken from patients prior to, and following cardioplegia during a coronary artery bypass grafting surgery $(\mathrm{CABG})$, which is a model of ischemia-reperfusion ${ }^{14}$. As sampling ventricular tissue in humans poses a risk for patients, tissues harvested from the right atrial appendage are used as a surrogate for the molecular events taking place in the myocardium as a whole. Participants were selected based on age, with young subjects classified as being under the age of 50 years, while aged subjects were over the age of 70 years. We hypothesized that the young cohort would have a pronounced autophagic response following post-operative reperfusion compared to the aged patients. This would be associated with a pro-survival response, and could provide insight into how age influences the mechanisms involved in the stress response following an ischemia-reperfusion insult.

\section{Methods}

Right atrial tissue sampling - Samples of right atria were taken immediately prior to and following cardioplegic cardiac arrest using a double purse-string approach, which is a well-established model of human myocardial ischemia-reperfusion injury ${ }^{15-17}$. The heart was arrested with high potassium $(27 \mathrm{mEq} / \mathrm{L})$ blood cardioplegia solution and was maintained with a low potassium formulation at $4^{\circ} \mathrm{C}$. After weaning from bypass and a brief period of reperfusion (5-15 minutes), the tissue between the two purse-string sutures was harvested. The pre-cardioplegic arrest samples wre taken pre-cardiopulmonary bypass (CPB) and pre-arrest at the time of venous cannulation. All tissues were flash frozen in liquid nitrogen. The average age of the young participants was 42.6 years, and all were male $(n=5)$. The average age of the aged participants was 73.8 years, and 10 of 11 were male $(\mathrm{n}=11)$. Patients were matched for co-morbidities including dyslipidemia and hypertension, as well as for prescribed medications. Participants were excluded if they had diabetes, kidney disease, cancer or any other underlying issues, and were excluded if they currently identified as smokers. Use of patient samples from the St. Michael's BioBank was approved by the Office of Research Ethics at York University (Certificate \#2018-114), as well as the Research and Ethics board at St. Michael's Hospital (REB 15-385).

Protein Extraction- Atrial samples were weighed and diluted in 10x Sakamoto buffer with protease and phosphatase inhibitors and lysed with a Tissue Lyser. Lysates were centrifuged at 14,000g for 10 minutes, and the supernate was stored at $-80^{\circ} \mathrm{C}$.

Immunoblotting- Atrial proteins were separated on polyacrylamide SDS-PAGE gels at $120 \mathrm{mV}$. Proteins were transferred onto nitrocellulose membranes and incubated in 5\% milk in wash buffer. Membranes were incubated with primary antibody overnight at $4^{\circ} \mathrm{C}$, and then with HRP-conjugated secondary antibodies for $1 \mathrm{hr}$ at room temperature the following day. Membranes were visualized with enhanced chemiluminesence. Image J software was used for quantifications.

Statistical Analyses- Unpaired t-tests were used to detect differences between young and aged subjects using GraphPad Prism 6.0. When comparing the effect ischemia-reperfusion (pre- and post-CABG) in the young and aged atria, a two-way ANOVA with repeated measures was used. The critical value was set at $\mathrm{p}<0.05$. 
All error bars represent the SEM.

Results

Mitochondrial markers following ischemia-reperfusion with age. No significant changes were found with age in the outer membrane protein VDAC, the inner membrane subunits COX I, and COX IV, as well as the matrix enzyme citrate synthase (Fig. 1 A-E). However, a $32 \%$ reduction in UQCRC2, a subunit of complex 3 of the electron transport chain (Fig. 1F; 0.74 vs 0.53 , main effect of age, $\mathrm{p}=0.03$ ), was observed with age, suggesting a compositional change that may be indicative of a complex III defect in the aging heart. This was also observed in a negative correlation between the UQCRC2 protein content and age (Fig. 1G; $\mathrm{r}=-0.61$, $\mathrm{p}=0.06)$.

IR resulted in $30 \%$ increases in VDAC ( 0.75 vs $1.05, p=0.03)$ and COX-I ( 0.63 vs $1.10, p=0.03)$, in a manner that was attenuated with age. This suggests an acute accumulation of mitochondrial proteins that are not being degraded.

Autophagy markers following ischemia-reperfusion with age. LC3 is a component of the autophagosomal membrane, which undergoes lipidation as part of its maturation from LC3-I to LC3-II. No significant changes were found in the immature or mature forms basally (Fig. 2B), or in the ratio of LC3II to LC3I (0.62 vs $1.27, \mathrm{p}=0.70$ ), despite a trend toward an increase with age (Fig. $2 \mathrm{C} ; 0.62$ vs $1.18, \mathrm{p}=0.82$ ). Furthermore, a negative correlation was seen between Optineurin, an autophagy adaptor protein, with age (Fig. 2G; r=-0.62, $\mathrm{p}=0.057)$. These data support an impairment in autophagy with age.

A decrease in Optineurin (main effect of IR; 1.14 vs $0.65, \mathrm{p}=0.003$ ) was also observed post-reperfusion in the young subjects, however this was not seen in the aged cohort (Fig. 2D). When accompanied by the marked reduction in the autophagy initiator Beclin-1 (Fig. 2F; main effect of IR, 0.93 vs $0.50, \mathrm{p}=0.001$ ), and the lack of change in $\mathrm{p} 62$ (2.03 vs $1.54, \mathrm{p}=0.10)$, these data suggest that the autophagy pathway was reduced post-IR.

Mitophagy markers following ischemia-reperfusion with age.Parkin, the E3 ubiquitin ligase, was reduced in aged atrial samples (Fig. 3D; $\mathrm{r}=-0.78, \mathrm{p}=0.02$ ), along with NIX, a receptor on the outer mitochondrial membrane that has roles in both mitophagy and in apoptosis. Following IR, Parkin levels were dramatically reduced in atrial samples of younger patients (Fig. 3B; 0.95 vs n.d., $p=0.02$ ), an effect that was not observed in the aged cohort (Fig. 3B; 0.49 vs 0.47 , interaction effect, $\mathrm{p}=0.04$; main effect of $\mathrm{IR}, \mathrm{p}=0.02$ ). This suggests a decline in mitophagy following IR in younger patients. However, this could have been compensated for by the elevation in NIX, by 3.8-fold in the young cohort ( 0.60 vs $1.8, \mathrm{p}=0.002$ ), but only 2.3 -fold in the aged subjects (Fig. 3C; 0.21 vs 0.76 , interaction effect, $\mathrm{p}=0.04$; main effect of IR, $\mathrm{p}=0.002$; main effect of age, $\mathrm{p}=0.004$ ). These data highlight a blunted response in the aged cohort to the IR injury, suggesting a loss in the ability to mount an appropriate response to stress with age.

Lysosomal markers following IR with age. Dramatic $68 \%$ and $85 \%$ reductions in TFEB and TFE3, the transcription factors that regulate lysosomal- and autophagy-related genes, were observed with age (Fig. 4BC; 0.63 vs $0.20, p=0.02 ; 1.00$ vs $0.10, p=0.0001$ respectively), which corresponded to negative correlations with age (Fig. 4F-G; r=-0.56, $\mathrm{p}=0.15 ; \mathrm{r}=-0.95, \mathrm{p}=0.001$ respectively). Furthermore, a trend for reduced Cathepsin-D, a lysosomal protease, was found in the aged cohort (Fig. $4 \mathrm{D} ; 0.54$ vs $0.34, \mathrm{p}=0.14$ ), but no change was observed for V-ATPase, a proton pump found on the lysosomal membrane (Fig. 4E; 0.51 vs 0.54). TFEB and TFE3 were markedly reduced in the young patients following IR (Fig. 4B-C; 0.63 vs n.d., main effect of IR, $\mathrm{p}=0.02$ for TFEB). Surprisingly, there was an increase in V-ATPase protein content in the atria of younger patients following IR (Fig. $4 \mathrm{E} ; 0.51$ vs 1.12 , main effect of IR, $\mathrm{p}=0.005$ ). These changes were dependent on age. TFEB content remained unchanged (Fig. 4B; 0.20 vs 0.25 , interaction effect, $\mathrm{p}=0.02$ ), while TFE3 content increased 3-fold (Fig. 4C; 0.10 vs 0.45 , interaction effect, $\mathrm{p}=0.01$ ), while V-ATPase did not respond in the aged cohort (Fig. $4 \mathrm{E} ; 0.54$ vs 0.71 , interaction effect, $\mathrm{p}=0.05$ ), supporting the notion of a blunted stress response in the atria of aged subjects, compared to the young (1.12 vs $0.71, \mathrm{p}=0.004)$. This would also support declines in the capacity for autophagy in the aged, which was evident by the reduced response in autophagy markers in the aged patients. 
Stress response markers following ischemia-reperfusion with age.No significant changes were observed in either HSP70 or caspase-3 with age, or following the IR (Fig. 5A-D).

\section{Conclusion}

The aging myocardium is characterized by a number of structural, biochemical and metabolic changes that contribute to a decline in cardiac function ${ }^{18}$. An emerging characteristic of the aging phenotype is a decline in autophagy, described in a number of tissues ${ }^{19,20}$. This is a significant alteration as autophagy is often cytoprotective and has been linked to improved outcomes in response to IR injuries ${ }^{3,11,21-23}$. Autophagy is also emerging as a quintessential mechanism involved in the protective benefits of ischemic preconditioning ${ }^{5,24}$, therapeutic hypothermia ${ }^{4}$ and a number of pharmaceuticals, such as statins ${ }^{25}$. Previous studies have highlighted a reliance on autophagy in the response to IR in patients undergoing cardioplegia during surgery ${ }^{15,22}$. The goal of this study was to contribute to our understanding of how age influences the autophagy response to IR insults in human cardiac tissue by using samples from right atrial appendages of patients undergoing CABG surgery.

Our results revealed no overt changes with age in several markers indicative of mitochondrial content, with the notable exception of a significant decrease in UQCRC2, a subunit of complex III. This is in line with previous reports of functional impairments in complex III activity in rodent models of aging ${ }^{2,26}$, and is suggestive of a change in mitochondrial composition with age.

The critical end-stage steps of the autophagy pathway reside within lysosomes, which serve to degrade dysfunctional cargo. Previous accounts have described evidence of lysosomal dysfunction with age, as these organelles tend to accumulate lipofuscin, indicative of a catabolic blockade ${ }^{27-29}$. Strikingly, we observed sharp declines in both TFEB and TFE3, transcriptional regulators of lysosomal biogenesis, with age. Despite this decline, only a trend for decrease in the lysosomal protein Cathepsin D was observed, a known target of TFEB. However, the induction of v-ATPase, a robust effect observed with IR in younger subjects, was completely blunted with age (Table 1). This suggests that TFEB and TFE3 levels may be sufficient to maintain basal levels of lysosomal proteins, but that the response to stress is severely restricted with age. Interestingly, a trend for an increase in the LC3 II:I ratio with age was observed without a change in the adaptor proteins p62 and Optineurin. Together, these data point to a decline in lysosomal function with age, since these proteins are normally consumed in the autophagy process, and an increase in LC3-II represents an accumulation of autophagosomes that have not fused to lysosomes for degradation. This would be in line with numerous studies that support a decrease in autophagy with age in other tissues, pointing potentially to reduced degradative capacity of the lysosome, rather than impairments at the initiation/tagging stages, or in the elongation of the phagophore ${ }^{11,22,30}$. Our data also suggest a decrease in mitophagy with age, evident from the marked decrements in both Parkin and Nix (Table 1). This could lead to the accumulation of mitochondria with altered composition, and possible dysfunction within the heart, evident from the decrease in UQCRC2. It is well known from other studies in muscle cells that the inhibition of lysosomal function leads to an accumulation of mitochondrial content, but that these organelles have reduced rates of respiration, as well as elevated ROS emission ${ }^{31}$. Future measures of mitochondrial function are required to verify this hypothesis.

Prior reports ${ }^{32}$ using the same surgical intervention have demonstrated that perioperative IR promotes mitochondrial biogenesis, likely by enhancing the translation of pre-existing mRNAs. This is in line with our observations of increased COX I and VDAC protein content. This increase in select mitochondrial markers may represent a protective response, aimed at maintaining cardiac metabolic capacity following an ischemic insult. It is important to note that not all mitochondrial markers increased following IR, and this may reflect a compositional change rather than an overall increase in content. Interestingly, the aged cohort exhibited a blunted increase in COX I protein content in comparison to young counterparts, further indicating an inability of the aged heart to mount an appropriate response to stress, as discussed above (Table 1). Increases in VDAC could also contribute to an enhanced activity of the mitochondrial permeability transition pore, and although increased pore formation to initiate mitochondrial-mediated apoptosis is a common finding with $\mathrm{IR}^{33-35}$, our data provide no evidence for enhanced apoptotic signaling following this IR intervention 
in this cohort.

The ubiquitination "tagging" of mitochondria could be impaired following IR in younger subjects, based on the marked decline in Parkin levels following this bypass surgery. The interpretation of impaired autophagy and mitophagy is supported by the modest trend for an increase in LC3-II protein content observed in the younger patients, along with previous accounts that have demonstrated poor clearance by the lysosomes in rodent models of IR. As the nature of the autophagy process is highly dynamic, researchers have relied on measurements of autophagy flux to better understand this recycling process, although many of these techniques rely on fluorescent tagging, or the use of inhibitors which would not be appropriate for human trials. Thus, it is critical to rely on data from animal models to fully appreciate the complexity of the IR response.

During enhanced autophagy, LC3-II and p62 should be degraded along with the dysfunctional cargo. The lack of decrease in these proteins, taken together with the decline in Beclin-1, which is involved in the initiation of autophagy, suggests that autophagy decreases following IR. TFEB and TFE3 also decline, and as transcriptional regulators of a number of autophagy-related genes ${ }^{36}$, these data also support this autophagy decrease. Interestingly, the postoperative response in these parameters appeared to be blunted in the aged cohort and this may be evidence of an inability to mount a stress response with age (Table 1).

In summary, our data support the idea that autophagy declines with age in human atria. It remains to be addressed whether this decrement lies in the initiation of the recycling process, the ability to detect and sequester damaged material, or in the capacity to breakdown cellular components at the lysosome. Understanding the autophagic response is also critical as it is thought to have repercussions for the activity of cell death or apoptotic pathways ${ }^{3,15,37}$. Our findings also demonstrate the influence of age on the response of the myocardium to surgeries involving ischemia-reperfusion, as older subjects exhibit a reduced resilience and adaptability compared to younger patients. Future work will be needed to resolve the relative roles of apoptosis and autophagy in the aging myocardium, and will be aided by measurements of mitochondrial function.

References

1. Sachs HG, Colgan JA, Lazarus ML. Ultrastructure of the aging myocardium: A morphometric approach. Am J Anat . 1977;150(1):63-71. doi:10.1002/aja.1001500105

2. Lemieux H, Vazquez EJ, Fujioka H, Hoppel CL. Decrease in mitochondrial function in rat cardiac permeabilized fibers correlates with the aging phenotype. Journals Gerontol - Ser A Biol Sci Med Sci . 2010;65 A(11):1157-1164. doi:10.1093/gerona/glq141

3. Ma X, Liu H, Foyil SR, et al. Impaired Autophagosome Clearance Contributes to Cardiomyocyte Death in Ischemia-Reperfusion Injury.Circulation . 2012;125(25):3170-3181. doi:10.1161/CIRCULATIONAHA.110.956839

4. Marek-Iannucci S, Thomas A, Hou J, et al. Myocardial hypothermia increases autophagic flux, mitochondrial mass and myocardial function after ischemia-reperfusion injury. Sci Rep . 2019;9(1):1-17. doi:10.1038/s41598-019-46452-w

5. Huang C, Yitzhaki S, Perry CN, et al. Autophagy induced by ischemic preconditioning is essential for cardioprotection. J Cardiovasc Transl Res . 2010;3(4):365-373. doi:10.1007/s12265-010-9189-3

6. Kanamori H, Takemura G, Goto K, et al. Autophagy limits acute myocardial infarction induced by permanent coronary artery occlusion.Am J Physiol - Hear Circ Physiol . 2011;300(6):2261-2271. doi:10.1152/ajpheart.01056.2010

7. Matsui Y, Takagi H, Qu X, et al. Distinct roles of autophagy in the heart during ischemia and reperfusion: Roles of AMP-activated protein kinase and beclin 1 in mediating autophagy. Circ Res . 2007;100(6):914-922. doi:10.1161/01.RES.0000261924.76669.36 
8. Ravikumar B, Berger Z, Vacher C, O'Kane CJ, Rubinsztein DC. Rapamycin pre-treatment protects against apoptosis. Hum Mol Genet . 2006;15(7):1209-1216. doi:10.1093/hmg/ddl036

9. Loos B, Genade S, Ellis B, Lochner A, Engelbrecht AM. At the core of survival: Autophagy delays the onset of both apoptotic and necrotic cell death in a model of ischemic cell injury. Exp Cell Res . 2011;317(10):14371453. doi:10.1016/j.yexcr.2011.03.011

10. Leidal AM, Levine B, Debnath J. Autophagy and the cell biology of age-related disease. Nat Cell Biol . 2018;20(12):1338-1348. doi:10.1038/s41556-018-0235-8

11. Taneike M, Yamaguchi O, Nakai A, et al. Inhibition of autophagy in the heart induces age-related cardiomyopathy. Autophagy . 2010;6(5):600-606. doi:10.4161/auto.6.5.11947

12. Nakai A, Yamaguchi O, Takeda T, et al. The role of autophagy in cardiomyocytes in the basal state and in response to hemodynamic stress. Nat Med . 2007;13(5):619-624. doi:10.1038/nm1574

13. Fernández ÁF, Sebti S, Wei Y, et al. Disruption of the beclin 1/Bcl-2 autophagy regulatory complex promotes longevity in mice.Nature . 2018;558(7708):136-140. doi:10.1038/s41586-018-0162-7.Disruption

14. Clements RT, Sodha NR, Feng J, et al. Phosphorylation and translocation of heat shock protein 27 and $\alpha \mathrm{B}$-crystallin in human myocardium after cardioplegia and cardiopulmonary bypass. J Thorac Cardiovasc Surg . 2007;134(6). doi:10.1016/j.jtcvs.2007.06.026

15. Singh KK, Yanagawa B, Quan A, et al. Autophagy gene fingerprint in human ischemia and reperfusion. J Thorac Cardiovasc Surg . 2014;147(3):1065-1072.e1. doi:10.1016/j.jtcvs.2013.04.042

16. Kleinbongard P, Gedik N, Kirca M, et al. Mitochondrial and contractile function of human right atrial tissue in response to remote ischemic conditioning. J Am Heart Assoc . 2018;7(15):1-17. doi:10.1161/JAHA.118.009540

17. Storti S, Vittorini S, Iascone M, et al. Analysis of the variation in the hsp70 and hsp90alpha mRNA expression in human myocardial tissue that has undergone surgical stress. Cell Stress Chaperones . $2003 ; 8(1): 18-25$.

18. Wilson PWF, Castelli WP, Kannel WB. Coronary risk prediction in adults (The Framingham Heart Study). Am J Cardiol . 1987;59(14). doi:10.1016/0002-9149(87)90165-2

19. Kiffin R, Kaushik S, Zeng M, et al. Altered dynamics of the lysosomal receptor for chaperone-mediated autophagy with age. J Cell Sci . 2007;120(5):782-791. doi:10.1242/jcs.001073

20. Barbosa MC, Grosso RA, Fader CM. Hallmarks of aging: An autophagic perspective. Front Endocrinol (Lausanne) . 2019;10(JAN):1-13. doi:10.3389/fendo.2018.00790

21. Pyo JO, Yoo SM, Ahn HH, et al. Overexpression of Atg5 in mice activates autophagy and extends lifespan. Nat Commun . 2013;4:1-9. doi:10.1038/ncomms3300

22. Jahania SM, Sengstock D, Vaitkevicius P, et al. Activation of the homeostatic intracellular repair response during cardiac surgery.J Am Coll Surg . 2013;216(4):719-726. doi:10.1016/j.jamcollsurg.2012.12.034

23. Yan Y, Finkel T. Autophagy as a regulator of cardiovascular redox homeostasis. Free Radic Biol Med . 2017;109(November 2016):108-113. doi:10.1016/j.freeradbiomed.2016.12.003

24. Huang C, Andres AM, Ratliff EP, Hernandez G, Lee P, Gottlieb RA. Preconditioning involves selective mitophagy mediated by parkin and p62/SQSTM1. PLoS One. 2011;6(6). doi:10.1371/journal.pone.0020975

25. Andres AM, Hernandez G, Lee P, et al. Mitophagy is required for acute cardioprotection by simvastatin. Antioxidants Redox Signal . 2014;21(14):1960-1973. doi:10.1089/ars.2013.5416

26. Lesnefsky EJ, Chen Q, Hoppel CL. Mitochondrial Metabolism in Aging Heart Edward. Circ Res . 2016;118(10):1593-1611. doi:10.1016/j.physbeh.2017.03.040 
27. Örlander J, Kiessling K-H, Larsson L, Karlsson J, Aniansson A. Skeletal muscle metabolism and ultrastructure in relation to age in sedentary men. Acta Physiol Scand . 1978;104(3):249-261. doi:10.1111/j.1748-1716.1978.tb06277.x

28. Höhn A, König J, Grune T. Protein oxidation in aging and the removal of oxidized proteins. J Proteomics . 2013. doi:10.1016/j.jprot.2013.01.004

29. Cuervo AM, Bergamini E, Bunk ET, Droge W, Ffrench M, Terman A. Autophagy and Aging: The Importance of Maintaining "Clean" Cells.Autophagy . 2005;1(3):131-140. doi:10.4161/auto.1.3.2017

30. Dutta D, Calvani R, Bernabei R, Leeuwenburgh C, Marzetti E. Contribution of impaired mitochondrial autophagy to cardiac aging: mechanisms and therapeutic opportunities. Circ Res . 2012;110(8):1125-1138. doi:10.1161/CIRCRESAHA.111.246108.Contribution

31. Parousis A, Carter HN, Tran C, et al. Contractile activity attenuates autophagy suppression and reverses mitochondrial defects in skeletal muscle cells. Autophagy . 2018;14(11):1886-1897. doi:10.1080/15548627.2018.1491488

32. Andres AM, Tucker KC, Thomas A, et al. Mitophagy and mitochondrial biogenesis in atrial tissue of patients undergoing heart surgery with cardiopulmonary bypass. JCI Insight . 2017;2(4). doi:10.1172/jci.insight.89303

33. Heusch G, Gersh BJ. The pathophysiology of acute myocardial infarction and strategies of protection beyond reperfusion: A continual challenge. Eur Heart J . 2017;38(11):774-784. doi:10.1093/eurheartj/ehw224

34. Baines CP. How and when do myocytes die during ischemia and reperfusion: The late phase. J Cardiovasc Pharmacol Ther . 2011;16(3-4):239-243. doi:10.1177/1074248411407769

35. Paradies G, Paradies V, Ruggiero FM, Petrosillo G. Mitochondrial bioenergetics and cardiolipin alterations in myocardial ischemia-reperfusion injury: Implications for pharmacological cardioprotection. Am $J$ Physiol - Hear Circ Physiol . 2018;315(5):H1341-H1352. doi:10.1152/ajpheart.00028.2018

36. Napolitano G, Ballabio A. TFEB at a glance. J Cell Sci . 2016;129:2475-2481.

37. Xie M, Kong Y, Tan W, et al. HDAC Inhibition Blunts Ischemia/Reperfusion Injury by Inducing Cardiomyocyte Autophagy.Circulation . 2014;129(10):1139-1151. doi:10.1161/CIRCULATIONAHA.113.002416.HDAC

Figure Legends:

Figure 1: Mitochondrial markers following ischemia-reperfusion with age. Representative western blots of mitochondrial markers pre- and post-operative in atrial samples of young and aged patients (A ). Graphical representation of changes in protein content of $\operatorname{VDAC}(\mathbf{B})$, citrate synthase $(\mathbf{C})$, COX I (D), COX IV $(\mathbf{E})$, and UQCRC2 $(\mathbf{F})$. Correlation between UQCRC2 protein expression and age $(\mathbf{G})$. Ponceau stain was used for normalization of the data. \#, effect of ischemia $\mathrm{p}<0.05$; *, effect of age $\mathrm{p}<0.05$; Y, young; A, aged; $\mathrm{n}=2-8$. VDAC, voltage dependent anion channel; COX I, cytochrome oxidase subunit I; COX IV, cytochrome oxidase subunit IV; UQCRC2, ubiquinol-cytochrome c reductase core protein 2.

Figure 2: Autophagy markers following ischemia-reperfusion with age. Representative blots of autophagy markers pre- and post-operative in atrial tissues of young and aged patients (A ). Graphical representation of changes in protein content of the mature form of LC3, LC3-II (B ), ratio of LC3-II to I (C), p62 (D ), Optineurin (E ), and Beclin-1 (F ). Correlation between age and Optineurin protein content based on western blot data $(\mathbf{G})$. Ponceau stain was used for normalization of the data. \#\#, effect of ischemia $\mathrm{p}<0.01$; Y, young; A, aged; n=2-11. LC3, microtubule-associated proteins 1A/1B light chain 3B; p62, sequestosome 1.

Figure 3 : Mitophagy markers following ischemia-reperfusion with age. Representative blots of mitophagy markers pre- and post-operative in atrial tissues of young and aged patients (A ). Graphical representation 
of observed changes in protein content of Parkin (B ), NIX (C ). Correlation between Parkin protein content and age $(\mathbf{G})$. Ponceau stain was used for normalization of the data. $\varphi$, interaction effect $\mathrm{p}<0.05$; \#, effect of ischemia $\mathrm{p}<0.05$; \#\#, effect of ischemia $\mathrm{p}<0.01$; **, effect of age $\mathrm{p}<0.01$; ***, effect of age $\mathrm{p}<0.001 ; \mathrm{Y}$, young; A, aged; $\mathrm{n}=2-11$.

Figure 4: Lysosomal markers following ischemia-reperfusion with age. Representative blots of lysosomal markers pre- and post-operative in atrial tissues of young and aged patients (A ). Graphical representation of changes in protein content of TFEB (B), TFE3 (C), immature Cathepsin D (D ), and v-ATPase (E ). Graph of the relationship between TFEB protein content $(\mathbf{F})$, TFE3 protein content $(\mathbf{G})$ and age. Ponceau stain was used for normalization of the data. $\varphi$, interaction effect $\mathrm{p}<0.05 ; \varphi \varphi$, interaction effect $\mathrm{p}<0.01$; \#, effect of ischemia $\mathrm{p}<0.05$; \#\#, effect of ischemia $\mathrm{p}<0.01$; ${ }^{*}$, effect of age $\mathrm{p}<0.05$; ${ }^{* *}$, effect of age $\mathrm{p}<0.01$; ***, effect of age $\mathrm{p}<0.001$; $\mathrm{Y}$, young; $\mathrm{A}$, aged; $\mathrm{n}=2-11$. TFEB, transcription factor EB; TFE3, transcription factor E3; v-ATPase, vacuolar-type $\mathrm{H}^{+}$ATPase.

Figure 5: Stress response markers following ischemia-reperfusion with age. Representative blots of stress response markers pre- and post-operative in atrial tissues of young and aged patients (A ). Graphical representation of changes in protein content of HSP70 (B ), Caspase-3 middle band (C), Caspase-3 top band (D ). Ponceau stain was used for normalization of the data. Y, young; A, aged; $n=5-11$. HSP70, heat shock protein 70 .

Table 1 : Differential responses to IR in young and aged patients. Summary of how proteins respond following IR in human atrial tissues and how age influences the overall stress response. This wide sampling of proteins illustrates a blunted response in the aged patients, which may influence the degree of recovery to IR.

\begin{tabular}{lll}
\hline & Young & Aged \\
\hline Mitochondrial Content & - VDAC & - VDAC \\
& - COX-I & N/C COX-I \\
Autophagy & - Beclin-1 & - Beclin-1 \\
& - LC3-II (trend) & N/C LC3-II \\
Mitophagy & - Optineurin & - Optineurin \\
Lysosomal Biogenesis & N/C Parkin \\
& - NIX & N/C NIX \\
& - TFE3 & N/C TFEB \\
& - v-ATPase & N/C v-ATPase \\
\hline
\end{tabular}

(N/C, no change)

\section{Hosted file}

SMH Figures -Mar 12 2021.pptx available at https://authorea.com/users/402086/articles/513984human-cardiac-ischemia-reperfusion-injury-blunted-stress-response-with-age 